\title{
ON WEAK CONTAINMENT PROPERTIES
}

\author{
HARALD RINDLER
}

(Communicated by William J. Davis)

\begin{abstract}
We prove, that two concepts of weak containment do not coincide, contradicting results in [1, Lemma 3.3 and Proposition 3.4]. The statement of Theorem 3.5 remains valid. There exist infinite tall compact groups $G$ (i.e. the set $\{\sigma \in \hat{G}, \operatorname{dim} \sigma=n\}$ is finite for each positive integer $n$ ) having the mean-zero weak containment property. Such groups do not have the dual Bohr approximation property or $A P(\hat{G}) \neq C_{\delta}^{*}(G)$.
\end{abstract}

Let $G$ be a compact group, then $G$ is said to have the mean-zero weak containment property if there exists a net

$$
\left\{g_{\alpha}\right\} \text { in } L_{0}^{2}(G)=\left\{f \in L^{2}(G), \int f(x) d x=0\right\}
$$

such that $\left\|g_{\alpha}\right\|_{2}=1$ and $\lim _{\alpha}\left\|L_{y} g_{\alpha}-g_{\alpha}\right\|_{2}=0$ for all $y \in G . L_{y}$ denotes the left translation operator, $L_{y} f(x)=f\left(y^{-1} x\right)$, see [4]. It was claimed in [1, Lemma 3.3], that this property were equivalent to the following weaker version: There exists $a$ net $h_{\alpha} \in L_{0}^{2}(G),\left\|h_{\alpha}\right\|_{2}=1$, such that $L_{y} h_{\alpha}-h_{\alpha} \rightarrow 0$ weakly in $L_{0}^{2}(G)$.

The statement of this lemma is false. We show, why the indicated proof does not work (the error in the proof of Lemma 3.3 [1] is not isolated in literature). Since for convex sets the weak closure coincides with the norm closure, we can replace $\left(h_{\alpha}\right)$ by some convex linear combinations $g_{\alpha}$ such that $\lim \left\|L_{y} g_{\alpha}-g_{\alpha}\right\|_{2}$ $=0, \forall y \in G$. But unfortunately it may happen, that $\lim \left\|g_{\alpha}\right\|_{2}=0$.

In fact, if $G$ is an infinite compact group, then $L_{0}^{2}(G)$ is infinite dimensional, therefore there exists a net $h_{\alpha} \in L_{0}^{2}(G)$, such that $\left\|h_{\alpha}\right\|_{2}=1$ and $\lim _{\alpha}\left\langle h_{\alpha}, f\right\rangle=$ 0 for all $f \in L_{0}^{2}(G)$, consequently

$$
\lim _{\alpha}\left\langle L_{y} h_{\alpha}-h_{\alpha}, f\right\rangle=\lim _{\alpha}\left\langle h_{\alpha}, L_{y-1} f\right\rangle-\lim _{\alpha}\left\langle h_{\alpha}, f\right\rangle=0
$$

for all $f \in L_{0}^{2}(G)$.

This has been proved in [1, Proposition 3.4] for $G=\prod_{n=1}^{\infty} G_{n}$ a direct product of nontrivial compact groups.

Received by the editors June $25,1990$.

1980 Mathematics Subject Classification (1985 Revision). Primary 22D10, 43A65.

Key words and phrases. Locally compact groups, left regular representation, amenable groups, Kazhdan's property $T$, tall groups. 
Remark 1. Example 1 shows that the mean-zero weak containment property does not hold for general infinite products of nontrivial compact groups as stated in [1, Proposition 3.4].

Example 1. If $n \geq 3, G=\prod_{p \in \mathbb{P}} S l\left(n, F_{p}\right), F_{p}=\mathbb{Z} / p \mathbb{Z}, \mathbb{P}$ denotes the set of all primes, then $G$ does not have the mean-zero weak containment property.

Proof. (The sketch of proof given in [3] is incomplete.) The canonical homomorphisms $\varphi_{p}: \mathbb{Z} \rightarrow \mathbb{Z} / p \mathbb{Z}=F_{p}$, define a homomorphism $\varphi: S l(n, \mathbb{Z}) \Rightarrow$ $\prod_{p \in \mathbb{P}} S l\left(n, \mathbb{Z}_{p}\right) \quad(n \geq 2) . \varphi$ is clearly injective (if $x \equiv y(\bmod p)$ for all $p \in \mathbb{P}$ then $x=y$ ). If we can show that the image of $\varphi$ is dense in $G$, then $G$ contains a dense subgroup isomorphic to $\operatorname{Sl}(n, \mathbb{Z})$, i.e. a group satisfying Kazhdan's property $T$, if $n \geq 3$ and consequently $G$ does not have the mean-zero weak containment property (cf. [5]). Therefore it suffices to proof

Lemma 1. Let $E$ be a finite set of primes, $A_{p}=\left(a_{i j, p}\right) \in S l(n, \mathbb{Z})$ such that $\operatorname{det} A_{p} \equiv 1(\bmod p)$ for all $p \in E$, then there exists $A=\left(a_{i j}\right) \in \operatorname{Sl}(n, \mathbb{Z})$ such that $a_{i j} \equiv a_{i j, p}(\bmod p)$ for all $p \in E$ and $1 \leq i, j \leq n$.

Proof. It suffices to consider the case $n=2$, since $\prod_{p \in E} S l\left(n, F_{p}\right)$ can be generated by nice subgroups $S l\left(2, F_{p}\right), p \in E$. Put $m=\prod_{p \in E} p$, then by the Chinese Remainder Theorem it follows that there exists $A=\left(a_{i j}\right), a_{i j} \equiv a_{i j, p}$ $(\bmod p)$ for all $p \in E, 1 \leq i, j \leq n$. We have $\operatorname{det} A \equiv \operatorname{det} A_{p} \equiv 1(\bmod p)$ for all $p \in E$, consequently $\operatorname{det} A=1+m x, x \in \mathbb{Z}$.

In particular $\left(a_{12}, a_{22}, m\right)=1$ (i.e. $a_{12}, a_{22}$ and $m$ have no nontrivial common divisors).

If $a_{i 2}=d_{i} b_{i}, d_{i}=\left(a_{i 2}, m\right)$, then $\left(d_{1}, d_{2}\right)=1$. Setting $\bar{a}_{i 2}=a_{i 2}+x_{i} m$ $\left(\equiv a_{i 2}(\bmod p)\right.$ for all $\left.p\right)$ we may obtain that $\left(\bar{b}_{1}, \bar{b}_{2}\right)=1$. (Dirichlet's prime number theorem implies even that we may assume that $\bar{b}_{1}, \bar{b}_{2}$ are distinct primes), or that $\left(\bar{a}_{12}, \bar{a}_{22}\right)=1$. Therefore there exist $y_{1}, y_{2} \in \mathbb{Z}$ such that $y_{1} \bar{a}_{22}-y_{2} \bar{a}_{12}=-x$. If $\bar{a}_{i 1}=a_{i 1}+y_{i} m, \bar{A}=\left(\bar{a}_{i j}\right)$, then $\operatorname{det} \bar{A}=\operatorname{det} A+$ $m\left(y_{1} \bar{a}_{22}-y_{2} \bar{a}_{12}\right)=1+m x-m x=1$. This concludes the proof of the lemma. Considering the "root subgroups" $U_{k l}$ instead of $H_{u v}$, the proof becomes easier, since in this case $A$ itself can be chosen to be a transvection and consequently $\operatorname{det} A=1$.

Remark 2. It can be easily seen that there always exist dense subgroups $H$ of $G=\prod_{n=1}^{\infty} G_{n}$ (e.g. $H=\bigoplus G_{n}$ weak direct sum) and nets $g_{\alpha}$ such that $\left\|g_{\alpha}\right\|_{2}=1$ and $\lim _{\alpha}\left\|L_{y} g_{\alpha}-g_{\alpha}\right\|_{2}=0, \forall y \in H$.

Remark 3. $G=\prod_{n=1}^{\infty} S_{n}, S_{n}$ the group of permutations of $n$ elements, gives an example of a nonamenable group $G$ having the mean-zero weak containment property, see [3, Example 5].

Remark 4. Let $d(H)$ denote the minimal number of generators of a finite group $H$. If $G=\prod_{n=1}^{\infty} H_{n}$, and $\lim \sup _{n \rightarrow \infty} d\left(H_{n}\right)=\infty$, then $G$ has the mean-zero weak containment property. (Any finite set $\left\{y_{1}, y_{2}, \ldots, y_{k}\right\}$ is contained in a subgroup $K=\prod_{n=1}^{\infty} K_{n}, K_{n}=H_{n}$ for $n \neq m, K_{m}=L_{m} \neq H_{m}$, if $d\left(H_{m}\right)>k$. If $g(x)=1$, if $x \notin K, g(x)=1-1 /[G: K]$, if $x \in K$, then $g \in L_{0}^{2}(G)$, $\|g\|_{2} \geq 1$ and $L_{y_{i}} g-g=0$ for $1 \leq i \leq k$.) Therefore we are able to prove:

Proposition 1. There exist compact tall groups $G$ having the mean-zero weak containment property. 
If $G_{n}$ is any sequence of pairwise nonisomorphic non-Abelian simple groups, then one can choose $G=\prod_{n=1}^{\infty} G_{n}^{k_{n}}$ for suitable $k_{n}$.

Proof. It follows from Hutchinson [2, Theorem 2.5] that any such groups are tall. Example 1 shows that in general one cannot take $k_{n}=1$ for all $n$. I learned from Kowol that $d(G)$ is small for a lot of groups (e.g. $d(H)=2$ for any simple non-Abelian group $H)$. Clearly $d\left(H^{r}\right)>s$, if $H=\left\{h_{1}, h_{2}, \ldots, h_{m}\right\}, r>m^{s}$, since $a_{i}=\left(a_{i 1}, \ldots, a_{i r}\right), 1 \leq i \leq s$ cannot generate $H^{r}\left(a_{i j}=a_{i k}\right.$, for some $j \neq k$ and $1 \leq i \leq s)$. Therefore Proposition 1 easily follows from Remark 4.

Remark 5. Proposition 1 implies that there exist compact groups $G$ which do not have the dual Bohr approximation property, see [1, Theorem 3.5].

\section{REFERENCES}

1. C. Chou, Almost periodic operators in $V N(G)$, Trans. Amer. Math. Soc. 317 (1990), 229-253.

2. M. F. Hutchison, Tall profinite groups, Bull. Austral. Math. Soc. 18 (1978), 421-428.

3. H. Rindler, Groups of measure preserving transformations. II, Math. Z. 199 (1988), 581-588.

4. J. Rosenblatt, Translation-invariant linear forms on $L_{p}(G)$, Proc. Amer. Math. Soc. 94 (1985), 226-228.

5. K. Schmidt, Amenability, Kazhdan's property $T$, strong ergodicity and invariant means for ergodic group actions, Ergodic Theory Dynamical Systems 1 (1981), 223-236.

Institut für Mathematik der Universität Wien, Strudlhofgasse 4, A-1090 Wien, AUSTRIA 